UT-02-32

\title{
Generalized Ginsparg-Wilson algebra and index theorem on the lattice
}

\author{
Kazuo Fujikawa \\ Department of Physics, University of Tokyo \\ Bunkyo-ku,Tokyo 113, Japan
}

\begin{abstract}
Recent studies of the topological properties of a general class of lattice Dirac operators are reported. This is based on a specific algebraic realization of the Ginsparg-Wilson relation in the form $\gamma_{5}\left(\gamma_{5} D\right)+\left(\gamma_{5} D\right) \gamma_{5}=2 a^{2 k+1}\left(\gamma_{5} D\right)^{2 k+2}$ where $k$ stands for a non-negative integer. The choice $k=0$ corresponds to the commonly discussed Ginsparg-Wilson relation and thus to the overlap operator. It is shown that local chiral anomaly and the instanton-related index of all these operators are identical. The locality of all these Dirac operators for vanishing gauge fields is proved on the basis of explicit construction, but the locality with dynamical gauge fields has not been established yet. We suggest that the Wilsonian effective action is essential to avoid infrared singularities encountered in general perturbative analyses.
\end{abstract}

\section{Introduction}

Recent developments in the treatment of fermions in lattice gauge theory are based on a hermitian lattice Dirac operator $\gamma_{5} D$ which satisfies the Ginsparg-Wilson relation [1]

$$
\gamma_{5} D+D \gamma_{5}=2 a D \gamma_{5} D
$$

where the lattice spacing $a$ is utilized to make a dimensional consideration transparent, and $\gamma_{5}$ is a hermitian chiral Dirac matrix. An explicit example of the operator satisfying (1.1) and free of species doubling has been given by Neuberger. [2] The relation (1.1) led to an interesting analysis of the notion of index in lattice gauge theory.[3] This index theorem in turn led to a new form of chiral symmetry, and the chiral anomaly is obtained as a non-trivial Jacobian factor under this modified chiral transformation. [4] This chiral Jacobian is regarded as a lattice generalization of the continuum path integral. [5] The very detailed analyses of the lattice chiral Jacobian have been performed. [G] It is also possible to formulate the lattice index theorem in a manner analogous to the continuum index theorem. [7], [8], [9] An interesting chirality sum rule, which relates the number of zero modes to that of the heaviest states, has also been noticed.[10] See Refs. [11] for reviews of these developments. 
We have recently discussed the possible generalization of (1.1) and its implications. 12 To be specific, we have discussed a generalization of the algebra (1.1) in the form

$$
\gamma_{5}\left(\gamma_{5} D\right)+\left(\gamma_{5} D\right) \gamma_{5}=2 a^{2 k+1}\left(\gamma_{5} D\right)^{2 k+2}
$$

where $k$ stands for a non-negative integer and $k=0$ corresponds to the ordinary GinspargWilson relation. When one defines

$$
H \equiv \gamma_{5} a D
$$

(1.2) is rewritten as

$$
\gamma_{5} H+H \gamma_{5}=2 H^{2 k+2}
$$

or equivalently

$$
\Gamma_{5} H+H \Gamma_{5}=0
$$

where we defined

$$
\Gamma_{5} \equiv \gamma_{5}-H^{2 k+1}
$$

Note that both of $H$ and $\Gamma_{5}$ are hermitian operators.

It has been shown that all the good topological properties of the overlap operator [2] is retained in this generalization. [12, 13] The practical applications of this generalization are not known at this moment. We however mention the charactristic properties of this generalization: The spectrum near the continuum configuration is closer to that of continuum theory and the chiral symmetry breaking terms become more irrelevent in the continuum limit for $k \geq 1$. The operator however spreads over more lattice points for large $k$.

\section{Representation of the general algebra}

We first discuss a general representation of the algebraic relation (1.5). The relation (1.5) suggests that if

$$
H \phi_{n}=a \lambda_{n} \phi_{n}, \quad\left(\phi_{n}, \phi_{n}\right)=1
$$

with a real eigenvalue $a \lambda_{n}$ for the hermitian operator $H$, then

$$
H\left(\Gamma_{5} \phi_{n}\right)=-a \lambda_{n}\left(\Gamma_{5} \phi_{n}\right)
$$

Namely, the eigenvalues $\lambda_{n}$ and $-\lambda_{n}$ are always paired if $\lambda_{n} \neq 0$ and $\left(\Gamma_{5} \phi_{n}, \Gamma_{5} \phi_{n}\right) \neq 0$. We also note the relation, which is derived by sandwiching the relation (1.4) by $\phi_{n}$,

$$
\left(\phi_{n}, \gamma_{5} \phi_{n}\right)=\left(a \lambda_{n}\right)^{2 k+1} \quad \text { for } \quad \lambda_{n} \neq 0
$$

Consequently

$$
\left|\left(a \lambda_{n}\right)^{2 k+1}\right|=\left|\left(\phi_{n}, \gamma_{5} \phi_{n}\right)\right| \leq\left\|\phi_{n}|||| \gamma_{5} \phi_{n}\right\|=1 .
$$

Namely, all the possible eigenvalues are bounded by

$$
\left|\lambda_{n}\right| \leq \frac{1}{a}
$$


We thus evaluate the norm of $\Gamma_{5} \phi_{n}$

$$
\begin{aligned}
\left(\Gamma_{5} \phi_{n}, \Gamma_{5} \phi_{n}\right) & =\left(\phi_{n},\left(\gamma_{5}-H^{2 k+1}\right)\left(\gamma_{5}-H^{2 k+1}\right) \phi_{n}\right) \\
& =\left(\phi_{n},\left(1-H^{2 k+1} \gamma_{5}-\gamma_{5} H^{2 k+1}+H^{2(2 k+1)}\right) \phi_{n}\right)
\end{aligned}
$$

where we used (2.3). By remembering that all the eigenvalues are real, we find that $\phi_{n}$ is a "highest" state

$$
\Gamma_{5} \phi_{n}=0
$$

only if

$$
\left[1-\left(a \lambda_{n}\right)^{2}\right]=\left(1-a \lambda_{n}\right)\left(1+a \lambda_{n}\right)=0
$$

for the Euclidean positive definite inner product $\left(\phi_{n}, \phi_{n}\right) \equiv \sum_{x} \phi_{n}^{\dagger}(x) \phi_{n}(x)$.

We thus conclude that the states $\phi_{n}$ with $\lambda_{n}= \pm \frac{1}{a}$ are not paired by the operation $\Gamma_{5} \phi_{n}$ and

$$
\gamma_{5} D \phi_{n}= \pm \frac{1}{a} \phi_{n}, \quad \gamma_{5} \phi_{n}= \pm \phi_{n}
$$

respectively. These eigenvalues are in fact the maximum or minimum of the possible eigenvalues of $H / a$ due to (2.5).

As for the vanishing eigenvalues $H \phi_{n}=0$, we find from (1.4) that $H \gamma_{5} \phi_{n}=0$, namely, $H\left[\left(1 \pm \gamma_{5}\right) / 2\right] \phi_{n}=0$. We can thus choose

$$
\gamma_{5} D \phi_{n}=0, \quad \gamma_{5} \phi_{n}=\phi_{n} \quad \text { or } \quad \gamma_{5} \phi_{n}=-\phi_{n}
$$

To summarize the analyses so far, all the normalizable eigenstates $\phi_{n}$ of $\gamma_{5} D=H / a$ are categorized into the following 3 classes:

(i) $n_{ \pm}$("zero modes"),

$$
\gamma_{5} D \phi_{n}=0, \quad \gamma_{5} \phi_{n}= \pm \phi_{n}
$$

(ii) $N_{ \pm}$("highest states"),

$$
\gamma_{5} D \phi_{n}= \pm \frac{1}{a} \phi_{n}, \quad \gamma_{5} \phi_{n}= \pm \phi_{n}, \quad \text { respectively }
$$

(iii) "paired states" with $0<\left|\lambda_{n}\right|<1 / a$,

$$
\gamma_{5} D \phi_{n}=\lambda_{n} \phi_{n}, \quad \gamma_{5} D\left(\Gamma_{5} \phi_{n}\right)=-\lambda_{n}\left(\Gamma_{5} \phi_{n}\right) .
$$

Note that $\Gamma_{5}\left(\Gamma_{5} \phi_{n}\right) \propto \phi_{n}$ for $0<\left|\lambda_{n}\right|<1 / a$.

We thus obtain the index relation [3, 4]

$$
\begin{aligned}
\operatorname{Tr} \Gamma_{5} & \equiv \sum_{n}\left(\phi_{n}, \Gamma_{5} \phi_{n}\right) \\
& =\sum_{\lambda_{n}=0}\left(\phi_{n}, \Gamma_{5} \phi_{n}\right)+\sum_{0<\left|\lambda_{n}\right|<1 / a}\left(\phi_{n}, \Gamma_{5} \phi_{n}\right)+\sum_{\left|\lambda_{n}\right|=1 / a}\left(\phi_{n}, \Gamma_{5} \phi_{n}\right)
\end{aligned}
$$




$$
\begin{aligned}
& =\sum_{\lambda_{n}=0}\left(\phi_{n}, \Gamma_{5} \phi_{n}\right) \\
& =\sum_{\lambda_{n}=0}\left(\phi_{n},\left(\gamma_{5}-H^{2 k+1}\right) \phi_{n}\right) \\
& =\sum_{\lambda_{n}=0}\left(\phi_{n}, \gamma_{5} \phi_{n}\right) \\
& =n_{+}-n_{-}=\text {index }
\end{aligned}
$$

where $n_{ \pm}$stand for the number of normalizable zero modes with $\gamma_{5} \phi_{n}= \pm \phi_{n}$ in the classification (i) above. We here used the fact that $\Gamma_{5} \phi_{n}=0$ for the "highest states" and that $\phi_{n}$ and $\Gamma_{5} \phi_{n}$ are orthogonal to each other for $0<\left|\lambda_{n}\right|<1 / a$ since they have eigenvalues with opposite signatures.

On the other hand, the relation $\operatorname{Tr} \gamma_{5}=0$, which is expected to be valid in (finite) lattice theory, leads to ( by using (2.3))

$$
\begin{aligned}
\operatorname{Tr} \gamma_{5} & =\sum_{n}\left(\phi_{n}, \gamma_{5} \phi_{n}\right) \\
& =\sum_{\lambda_{n}=0}\left(\phi_{n}, \gamma_{5} \phi_{n}\right)+\sum_{\lambda_{n} \neq 0}\left(\phi_{n}, \gamma_{5} \phi_{n}\right) \\
& =n_{+}-n_{-}+\sum_{\lambda_{n} \neq 0}\left(a \lambda_{n}\right)^{2 k+1}=0 .
\end{aligned}
$$

In the last line of this relation, all the states except for the "highest states" with $\lambda_{n}= \pm 1 / a$ cancel pairwise for $\lambda_{n} \neq 0$. We thus obtain a chirality sum rule[10]

$$
n_{+}+N_{+}=n_{-}+N_{-}
$$

where $N_{ \pm}$stand for the number of "highest states" with $\gamma_{5} \phi_{n}= \pm \phi_{n}$ in the classification (ii) above. These relations show that the chirality asymmetry at vanishing eigenvalues is balanced by the chirality asymmetry at the largest eigenvalues with $\left|\lambda_{n}\right|=1 / a$. It was argued in Ref.[14] that $N_{ \pm}$states are the topological (instanton-related) excitations of the would-be species doublers.

We have thus established that the representation of all the algebraic relations (1.2) has a similar structure. In the next section, we show that the index $n_{+}-n_{-}$is identical to all these algebraic relations if the operator $\gamma_{5} D$ satisfies suitable conditions.

\section{Chiral Jacobian and the index relation}

The Euclidean path integral for a fermion is defined by

$$
\int \mathcal{D} \bar{\psi} \mathcal{D} \psi \exp \left[\int \bar{\psi} D \psi\right]
$$

where

$$
\int \bar{\psi} D \psi \equiv \sum_{x, y} \bar{\psi}(x) D(x, y) \psi(y)
$$


and the summation runs over all the points on the lattice. The relation (1.5) is re-written as

$$
\gamma_{5} \Gamma_{5} \gamma_{5} D+D \Gamma_{5}=0
$$

and thus the Euclidean action is invariant under the global "chiral" transformation [ـ]

$$
\begin{aligned}
& \bar{\psi}(x) \rightarrow \bar{\psi}^{\prime}(x)=\bar{\psi}(x)+i \sum_{z} \bar{\psi}(z) \epsilon \gamma_{5} \Gamma_{5}(z, x) \gamma_{5} \\
& \psi(y) \rightarrow \psi^{\prime}(y)=\psi(y)+i \sum_{w} \epsilon \Gamma_{5}(y, w) \psi(w)
\end{aligned}
$$

with an infinitesimal constant parameter $\epsilon$. Under this transformation, one obtains a Jacobian factor

$$
\mathcal{D} \bar{\psi}^{\prime} \mathcal{D} \psi^{\prime}=J \mathcal{D} \bar{\psi} \mathcal{D} \psi
$$

with

$$
J=\exp \left[-2 i \operatorname{Tr} \epsilon \Gamma_{5}\right]=\exp \left[-2 i \epsilon\left(n_{+}-n_{-}\right)\right]
$$

where we used the index relation (2.14).

We now relate this index appearing in the Jacobian to the Pontryagin index of the gauge field in a smooth continuum limit by following the procedure in Ref. [12]. We start with

$$
\operatorname{Tr}\left\{\Gamma_{5} f\left(\frac{\left(\gamma_{5} D\right)^{2}}{M^{2}}\right)\right\}=\operatorname{Tr}\left\{\Gamma_{5} f\left(\frac{(H / a)^{2}}{M^{2}}\right)\right\}=n_{+}-n_{-}
$$

Namely, the index is not modified by any regulator $f(x)$ with $f(0)=1$ and $f(x)$ rapidly going to zero for $x \rightarrow \infty$, as can be confirmed by using (2.14). This means that you can use any suitable $f(x)$ in the evaluation of the index by taking advantage of this property.

We then consider a local version of the index

$$
\operatorname{tr}\left\{\Gamma_{5} f\left(\frac{\left(\gamma_{5} D\right)^{2}}{M^{2}}\right)\right\}(x, x)=\operatorname{tr}\left\{\left(\gamma_{5}-H^{2 k+1}\right) f\left(\frac{\left(\gamma_{5} D\right)^{2}}{M^{2}}\right)\right\}(x, x)
$$

where trace stands for Dirac and Yang-Mills indices; Tr in (3.7) includes a sum over the lattice points $x$. A local version of the index is not sensitive to the precise boundary condition, and one may take an infinite volume limit of the lattice in the above expression.

We now examine the continuum limit $a \rightarrow 0$ of the above local expression (3.8)円. We first observe that the term

$$
\operatorname{tr}\left\{H^{2 k+1} f\left(\frac{\left(\gamma_{5} D\right)^{2}}{M^{2}}\right)\right\}
$$

goes to zero in this limit. The large eigenvalues of $H=a \gamma_{5} D$ are truncated at the value $\sim a M$ by the regulator $f(x)$ which rapidly goes to zero for large $x$. In other words, the global index of the operator $\operatorname{Tr} H^{2 k+1} f\left(\frac{\left(\gamma_{5} D\right)^{2}}{M^{2}}\right) \sim O(a M)^{2 k+1} \rightarrow 0$ for $a \rightarrow 0$ with fixed $M$.

We thus examine the small $a$ limit of

$$
\operatorname{tr}\left\{\gamma_{5} f\left(\frac{\left(\gamma_{5} D\right)^{2}}{M^{2}}\right)\right\}
$$

\footnotetext{
${ }^{1}$ This continuum limit corresponds to the so-called "naive" continuum limit in the context of lattice gauge theory.
} 
The operator appearing in this expression is well regularized by the function $f(x)$, and we evaluate the above trace by using the plane wave basis to extract an explicit gauge field dependence. We consider a square lattice where the momentum is defined in the Brillouin zone

$$
-\frac{\pi}{2 a} \leq k_{\mu}<\frac{3 \pi}{2 a}
$$

We assume that the operator $D$ is free of species doubling, which is proved for the explicit construction of $D$; in other words, the operator $D$ blows up rapidly $\left(\sim \frac{1}{a}\right)$ for small $a$ in the momentum region corresponding to species doublers. The contributions of doublers are eliminated by the regulator $f(x)$ in the above expression, since

$$
\operatorname{tr}\left\{\gamma_{5} f\left(\frac{\left(\gamma_{5} D\right)^{2}}{M^{2}}\right)\right\} \sim\left(\frac{1}{a}\right)^{4} f\left(\frac{1}{(a M)^{2}}\right) \rightarrow 0
$$

for $a \rightarrow 0$ if one chooses $f(x)=e^{-x}$, for example.

We thus examine the above trace in the momentum range of the physical species

$$
-\frac{\pi}{2 a} \leq k_{\mu}<\frac{\pi}{2 a}
$$

We obtain the limiting $a \rightarrow 0$ expression

$$
\begin{aligned}
& \lim _{a \rightarrow 0} \operatorname{tr}\left\{\gamma_{5} f\left(\frac{\left(\gamma_{5} D\right)^{2}}{M^{2}}\right)\right\}(x, x) \\
= & \lim _{a \rightarrow 0} \operatorname{tr} \int_{-\frac{\pi}{2 a}}^{\frac{\pi}{2 a}} \frac{d^{4} k}{(2 \pi)^{4}} e^{-i k x} \gamma_{5} f\left(\frac{\left(\gamma_{5} D\right)^{2}}{M^{2}}\right) e^{i k x} \\
= & \lim _{L \rightarrow \infty} \lim _{a \rightarrow 0} \operatorname{tr} \int_{-L}^{L} \frac{d^{4} k}{(2 \pi)^{4}} e^{-i k x} \gamma_{5} f\left(\frac{\left(\gamma_{5} D\right)^{2}}{M^{2}}\right) e^{i k x} \\
= & \lim _{L \rightarrow \infty} \operatorname{tr} \int_{-L}^{L} \frac{d^{4} k}{(2 \pi)^{4}} e^{-i k x} \gamma_{5} f\left(\frac{\left(i \gamma_{5} \not D\right)^{2}}{M^{2}}\right) e^{i k x} \\
\equiv & \operatorname{tr}\left\{\gamma_{5} f\left(\frac{\not D^{2}}{M^{2}}\right)\right\}
\end{aligned}
$$

where we first take the limit $a \rightarrow 0$ with fixed $k_{\mu}$ in $-L \leq k_{\mu} \leq L$, and then take the limit $L \rightarrow \infty$. This procedure is justified if the integral is well convergent. [12 We also assumed that the operator $D$ satisfies the following relation in the limit $a \rightarrow 0$

$$
\begin{aligned}
D e^{i k x} h(x) & \rightarrow e^{i k x}(-\not \not+i \not \partial-g \not A) h(x) \\
& =i(\not \partial+i g \not A)\left(e^{i k x} h(x)\right) \equiv i \not D\left(e^{i k x} h(x)\right)
\end{aligned}
$$

for any fixed $k_{\mu},\left(-\frac{\pi}{2 a}<k_{\mu}<\frac{\pi}{2 a}\right)$, and a sufficiently smooth function $h(x)$. The function $h(x)$ corresponds to the gauge potential in our case, which in turn means that the gauge potential $A_{\mu}(x)$ is assumed to vary very little over the distances of the elementary lattice spacing. 
Our final expression (3.14) in the limit $M \rightarrow \infty$ reproduces the Pontryagin number in the continuum formulation (with $\epsilon^{1234}=1$ ) [5

$$
\begin{gathered}
\lim _{M \rightarrow \infty} \operatorname{tr} \gamma_{5} f\left(\not D^{2} / M^{2}\right)=\operatorname{tr}_{5} \frac{1}{2 !}\left\{\frac{i g}{4}\left[\gamma^{\mu}, \gamma^{\nu}\right] F_{\mu \nu}\right\}^{2} \int \frac{d^{4} k}{(2 \pi)^{4}} f^{\prime \prime}\left(-k_{\mu} k^{\mu}\right) \\
=\frac{g^{2}}{32 \pi^{2}} \operatorname{tr} \epsilon^{\mu \nu \alpha \beta} F_{\mu \nu} F_{\alpha \beta}
\end{gathered}
$$

When one combines (3.7) and (3.16), one reproduces the Atiyah-Singer index theorem (in continuum $R^{4}$ space) . [7, 8] We note that a local version of the index (anomaly) is valid for Abelian theory also. The global index (3.7) as well as a local version of the index (3.8) are both independent of the regulator $f(x)$ provided [5]

$$
f(0)=1, \quad f(\infty)=0,\left.\quad f^{\prime}(x) x\right|_{x=0}=\left.f^{\prime}(x) x\right|_{x=\infty}=0 .
$$

We have thus established that the lattice index in (3.7) for any algebraic relation in (1.2) is related to the Pontryagin index in a smooth continuum limit as

$$
n_{+}-n_{-}=\int d^{4} x \frac{g^{2}}{32 \pi^{2}} \operatorname{tr} \epsilon^{\mu \nu \alpha \beta} F_{\mu \nu} F_{\alpha \beta}
$$

This shows that the instanton-related topological property is identical for all the algebraic relations in (1.2), and the Jacobian factor (3.6) in fact contains the correct chiral anomaly. (We are implicitly assuming that the index (3.7) does not change in the process of taking a continuum limit.)

A detailed perturbative analysis of chiral anomaly for the general operators with $k>0$ has been performed, and the above result has been confirmed. [12] Also a numerical study

of the index relation has been performed: The numerical result indicates the consistency of our analyses. [13]

\section{Explicit construction of the lattice Dirac operator for $k>1$}

We now comment on an explicit construction of the lattice Dirac operator which satisfies the generalized algebraic relation (1.2) with $k>0$. We start with the conventional Wilson fermion operator $D_{W}$ defined by

$$
\begin{aligned}
D_{W}(x, y) & \equiv i \gamma^{\mu} C_{\mu}(x, y)+B(x, y)-\frac{1}{a} m_{0} \delta_{x, y}, \\
C_{\mu}(x, y) & =\frac{1}{2 a}\left[\delta_{x+\hat{\mu} a, y} U_{\mu}(y)-\delta_{x, y+\hat{\mu} a} U_{\mu}^{\dagger}(x)\right], \\
B(x, y) & =\frac{r}{2 a} \sum_{\mu}\left[2 \delta_{x, y}-\delta_{y+\hat{\mu} a, x} U_{\mu}^{\dagger}(x)-\delta_{y, x+\hat{\mu} a} U_{\mu}(y)\right], \\
U_{\mu}(y) & =\exp \left[i a g A_{\mu}(y)\right],
\end{aligned}
$$


where we added a constant mass term to $D_{W}$ for later convenience. The parameter $r$ stands for the Wilson parameter. Our matrix convention is that $\gamma^{\mu}$ are anti-hermitian, $\left(\gamma^{\mu}\right)^{\dagger}=-\gamma^{\mu}$, and thus $\phi \equiv \gamma^{\mu} C_{\mu}(n, m)$ is hermitian

$$
\phi^{\dagger}=\varnothing
$$

The Dirac operator for a general value of $k$ is constructed by rewriting (1.2) as a set of relations

$$
\begin{aligned}
& H^{2 k+1} \gamma_{5}+\gamma_{5} H^{2 k+1}=2 H^{2(2 k+1)}, \\
& H^{2} \gamma_{5}-\gamma_{5} H^{2}=0,
\end{aligned}
$$

with $H=a \gamma_{5} D$. The second relation in (4.3) is shown by using the defining relation (1.4), and the first of these relations (4.3) becomes identical to the ordinary Ginsparg-Wilson relation (1.1) if one defines $H_{(2 k+1)} \equiv H^{2 k+1}$. One can thus construct a solution to (4.3) by following the prescription used by Neuberger [2]

$$
H_{(2 k+1)}=\frac{1}{2} \gamma_{5}\left[1+D_{W}^{(2 k+1)} \frac{1}{\sqrt{\left(D_{W}^{(2 k+1)}\right)^{\dagger} D_{W}^{(2 k+1)}}}\right]
$$

where

$$
D_{W}^{(2 k+1)} \equiv i(\varphi)^{2 k+1}+B^{2 k+1}-\left(\frac{m_{0}}{a}\right)^{2 k+1}
$$

The operator $H$ itself is then finally defined by (in the representation where $H_{(2 k+1)}$ is diagonal)

$$
H=\left(H_{(2 k+1)}\right)^{1 / 2 k+1}
$$

in such a manner that the second relation of (4.3) is satisfied. This condition (4.3) is shown to be satisfied in the representation where $H_{(2 k+1)}$ is diagonal. [12] Also the conditions $0<m_{0}<2 r=2$ and

$$
2 m_{0}^{2 k+1}=1
$$

ensure the absence of species doublers and a proper normalization of the Dirac operator $H$.

\section{$5 \quad$ Locality properties of general operators}

We have explained that the general operators for any finite $k$ give rise to correct chiral anomaly and index relations in the (naive) continuum limit. This suggests that those operators are local for sufficiently smooth background gauge field configurations. The locality of the standard overlap operator with $k=0$ has been established by Hernandez, Jansen and Lüscher, 15 and by Neuberger. 16.

As for the direct proof of locality of the operator $D$ for general $k$, one can show it for the vanishing gauge field by using the explicit solution for the operator $H$ in momentum 
representation [12, 13]

$$
\begin{aligned}
H\left(a p_{\mu}\right) & =\gamma_{5}\left(\frac{1}{2}\right)^{\frac{k+1}{2 k+1}}\left(\frac{1}{\sqrt{H_{W}^{2}}}\right)^{\frac{k+1}{2 k+1}}\left\{\left(\sqrt{H_{W}^{2}}+M_{k}\right)^{\frac{k+1}{2 k+1}}-\left(\sqrt{H_{W}^{2}}-M_{k}\right)^{\frac{k}{2 k+1}} \frac{\mathscr{s}}{a}\right\} \\
& =\gamma_{5}\left(\frac{1}{2}\right)^{\frac{k+1}{2 k+1}}\left(\frac{1}{\sqrt{F_{(k)}}}\right)^{\frac{k+1}{2 k+1}}\left\{\left(\sqrt{F_{(k)}}+\tilde{M}_{k}\right)^{\frac{k+1}{2 k+1}}-\left(\sqrt{F_{(k)}}-\tilde{M}_{k}\right)^{\frac{k}{2 k+1}} s\right\}
\end{aligned}
$$

where

$$
\begin{aligned}
F_{(k)} & =\left(s^{2}\right)^{2 k+1}+\tilde{M}_{k}^{2}, \\
\tilde{M}_{k} & =\left[\sum_{\mu}\left(1-c_{\mu}\right)\right]^{2 k+1}-m_{0}^{2 k+1}
\end{aligned}
$$

and

$$
\begin{aligned}
& s_{\mu}=\sin a p_{\mu} \\
& c_{\mu}=\cos a p_{\mu} \\
& \mathcal{s}^{\prime}=\gamma^{\mu} \sin a p_{\mu} .
\end{aligned}
$$

For $k=0$, this operator is reduced to Neuberger's overlap operator. [2] Here the inner product is defined to be $s^{2} \geq 0$. This operator is shown to be free of species doublers for the parameter $m_{0}$ within the range $0<m_{0}<2$ when we set $r=1$, and $2 m_{0}^{2 k+1}=1$ gives a proper normalization of $H$, namely, for an infinitesimal $p_{\mu}$, i.e., for $\left|a p_{\mu}\right| \ll 1$,

$$
H \simeq-\gamma_{5} a \not p\left(1+O(a p)^{2}\right)+\gamma_{5}\left(\gamma_{5} a \not p\right)^{2 k+2}
$$

to be consistent with $H=\gamma_{5} a D$; the last term in the rigth-hand side is the leading term of chiral symmetry breaking terms.

The locality of this explicit construction (5.1) is shown by studying the analytic properties in the Brillouin zone. [12] It is important to recognize that this operator is not ultra-local but exponentially local; 17 the operator $H(x, y)$ decays exponentially for large separation in coordinate representation

$$
H(x, y) \sim \exp [-|x-y| /(2.5 k a)] .
$$

An explicit analysis of the locality of the operator $H_{(2 k+1)}$ (not $H$ itself)in the presence of gauge field, in particular, the locality domain for the gauge field strength $\left\|F_{\mu \nu}\right\|$ has been performed. The locality domain for $\left\|F_{\mu \nu}\right\|$ becomes smaller for larger $k$, but a definite nonzero domain has been established. [12 The remaing task is to show the locality domain of $\left\|F_{\mu \nu}\right\|$ for the operator $H=\left(H_{(2 k+1)}\right)^{1 /(2 k+1)}$. Due to the operation of taking the $(2 k+1)$ th root, an explicit analysis has not been performed yet, though a supporting argument has been given. [12]. 


\section{Conclusion}

We have reported the recent investigation of topological properties of a general class of lattice Dirac operators defined by the algebraic relation (1.2). All these operators satisfy the index theorem and thus they are topologically proper. A precise proof of the locality of these general Dirac operators with fully dynamical gauge fields remains to be formulated. The operators with large $k$ is expected to exhibit infrared singularities in perturbative analyses as is suggested by the construction of $H_{(2 k+1)}$ in (4.4), and thus the Wilsonian formulation of effective action, which is supposed to be free of infrared singularities, would be essential.

Although we discussed only 4-dimensional theory, the recent developments in the treatment of lattice fermions 11 may have some implications on 2-dimensional theory also, which is the main subject of this Symposium. In this respect, the fact that the lattice Dirac operators are not ultra-local but exponentially local 15 may be of some interest. See Ref. [18 for a Ginsparg-Wilson construction on a 2-dimensional fuzzy sphere.

\section{Acknowledgments}

I thank Mo-lin Ge for the hospitality at Nankai University.

\section{References}

[1] P.H. Ginsparg and K.G. Wilson, Phys. Rev. D25 (1982)2649.

[2] H. Neuberger, Phys. Lett.B417(1998)141;B427(1998) 353.

[3] P. Hasenfratz, V. Laliena and F. Niedermayer, Phys. Lett. B427(1998)125, and references therein.

[4] M. Lüscher, Phys. Lett. B428(1998)342.

[5] K. Fujikawa, Phys. Rev. Lett. 42(1979)1195; Phys. Rev. D21 (1980)2848;D22(1980)1499(E).

[6] Y. Kikukawa and A. Yamada, Phys. Lett. B448(1999)265.

D.H. Adams, "Axial anomaly and topological charge in lattice gauge theory with overlap-Dirac", hep-lat/9812003.

H. Suzuki,Prog.Theor.Phys.102(1999)141.

K. Fujikawa,Nucl.Phys. B546(1999)480.

[7] R. Jackiw and C. Rebbi, Phys. Rev. D16(1977)1052.

[8] M. Atiyah, R. Bott, and V. Patodi, Invent. Math. 19(1973)279. 
[9] As for reviews of chiral anomaly in continuum theory,

S.L. Adler, in Lectures on Elementary Particles and Quantum Field Theory, edited by S. Deser et al. (MIT Press, Cambridge, Mass., 1970).

S.B. Treiman, R. Jackiw, B. Zumino and E. Witten, Current Algebra and Anomalies (World Scientific, Singapore, 1985).

R. Bertlmann, Anomalies in Quantum Field Theory (Oxford University Press, Oxford, 1996).

K. Fujikawa, "Aspects of anomalies in field theory", Int. J. Mod. Phys. A16 (2001) 331.

[10] T.W. Chiu, Phys. Rev. D58(1998)074511.

[11] As for reviews of recent developments in lattice fermions,

F. Niedermayer, Nucl. Phys, Proc. Suppl.73 (1999) 105.

H. Neuberger, "Exact chiral symmetry on the lattice," hep-lat/0101006.

M. Lüscher, "Chiral gauge theories revisited," hep-th/0102028.

[12] K. Fujikawa, Nucl. Phys. B589(2000)487.

K. Fujikawa and M. Ishibashi, Nucl. Phys. B587(2000)419.

K. Fujikawa and M. Ishibashi, Nucl. Phys. B605(2001) 365.

[13] T.W. Chiu, Nucl. Phys. B588 (2000) 400; Phys. Lett. 498B (2001) 111; Nucl. Phys. Proc. Suppl.94 (2001) 733.

[14] K. Fujikawa, Phys. Rev. D60(1999)074505.

[15] P. Hernandez, K. Jansen and M. Lüscher, Nucl. Phys. B552,363 (1999).

[16] H. Neuberger, Phys.Rev.D61(2000)085015.

[17] I. Horvath, Phys. Rev. Lett. 81 (1998) 4063.

[18] A. P. Balachandran, T. R. Govindarajan, and B. Ydri, hep-th/0006216. 